\title{
GENERATING COMPONENT RELEASE PLANS WITH BACKWARD SIMULATION
}

\author{
Edward F. Watson \\ Systems Modeling Corporation \\ The Park Building \\ 504 Beaver Street \\ Sewickley, PA 15143
}

\author{
Deborah J. Medeiros \\ Department of Industrial and \\ Management Systems Engineering \\ The Pennsylvania State University \\ 207 Hammond Building \\ University Park, PA 16802
}

\author{
Randall P. Sadowski \\ Systems Modeling Corporation \\ The Park Building \\ 504 Beaver Street \\ Sewickley, PA 15143
}

\begin{abstract}
Simulation modeling has traditionally been used in the discrete parts environment for facility design and capacity planning studies. More recently, simulationbased models have been used for generating dispatch lists in scheduling related activities. Currently, there is a growing interest to break away from viewing simulation narrowly as a predictive tool. This paper introduces the concept of backward simulation as a means of determining a required current state based on a desired goal state. This concept is developed into a procedure for generating component release plans based on a master production schedule. Details of this approach are presented.
\end{abstract}

\section{INTRODUCTION}

Manufacturers today must be more responsive to the rapidly changing marketplace in order to maintain a competitive edge. 'Quick response' (QR) is a term that describes the ability to respond quickly to diverse customer demand and to frequent changes to the product mix.. Achieving QR generally requires short product lead times and the flexibility to produce a diverse product mix. However, being responsive to demand in the marketplace is not the only challenge. Competition is forcing manufacturers to place an emphasis on finding creative ways to do more with less-less manpower, fewer resources, and less inventory. There is currently a tendency to shift away from long product life cycles, large batch sizes, and long process lead times.

To support the transition towards a QR environment, manufacturers are evolving from a make-to-stock environment to a make-to-order environment. This conversion demands not only a new way of controlling production, but also a new way of planning for production. The most common procedure known today for order release planning in a discrete parts environment is Material Requirements Planning (MRP). Perhaps the greatest benefit of MRP is its ability to determine independent demand requirements based on dependent demand. MRP, though, has received much criticism for its infinite capacity and planned lead time assumptions. That is, it relies on a Capacity Requirements Planning (CRP) module to verify capacity requirements. Furthermore, lead time estimates that are insensitive to shop conditions (e.g., load and capacity) and job characteristics (e.g., lot size and priority) are used to generate the component release plan. There appears to be an opportunity for a new tool for order release planning that is more sensitive to shop floor conditions and more responsive to diverse market demand.

Simulation modeling is a tool that has long been used for solving complex production-related problems, from long-term layout and capacity problems to short-term production scheduling problems [Swain, et. al., 1992; Nelson, et. al., 1991; Balci, et. al., 1990]. As depicted in Figure 1, each type of production problem addressed with simulation can be related to a time frame and some level of detail. More recently, simulation is being used to generate dispatch lists for operations scheduling applications and is also showing potential for shop floor control and real time monitoring applications. However, there is a growing interest to break away from viewing simulation narrowly as a way of making predictions by running an encoded behavioral model to answer "WhatIf" questions [Rothenberg, et. al., 1989].

Although it is accepted that certain planning activities are better addressed with alternative Operations Research tools (e.g., mathematical programming), it is felt that simulation may be used to bridge the gap between long term planning and short term scheduling. In this manner, a number of benefits may be gained. Naturally, if a simulation analyst has spent a major effort developing and validating a simulation model for a facility design application, the analyst may be particularly interested in 


\begin{tabular}{|c|c|c|c|c|c|}
\hline Year & $\begin{array}{l}\text { Facility Layout } \\
\text { Resource Planning }\end{array}$ & & & & \\
\hline Month & $\begin{array}{l}\text { Production Planning } \\
\text { Capacity Planning }\end{array}$ & Master Production & schedule & & \\
\hline Week & & & $\begin{array}{l}\text { MRP } \\
\text { CRP }\end{array}$ & & \\
\hline Day & & & & $\begin{array}{l}\text { Finite Capacity Sch } \\
\text { Operation Scheduli }\end{array}$ & eduling \\
\hline $\begin{array}{l}\text { Production } \\
\text { Shift }\end{array}$ & & & & & $\begin{array}{l}\text { Shop Floor Control } \\
\text { and Monitor }\end{array}$ \\
\hline
\end{tabular}

Figure 1: Hierarchy of Planning and Scheduling Problems

re-using the same model for other purposes [Davis, et. al., 1990].

In this paper, a methodology for planning and scheduling production based on backward and forward simulations is presented. Backward simulation is integrated with the fundamental dependent demand concepts of MRP, resulting in a planning technique that is forced to make realistic assumptions about the shop floor during the planning process. This approach should improve the planning process and alleviate the burden on the scheduling activity.

\section{BACKWARD APPROACH}

At the root of all problem solving is the concept of search. The basic objective of search is to reach a final (desired) state from some initial state. In forward search, the initial state is the current state and the desired state is the destination state. In backward search, the initial state is assumed to be the destination state and the final state is the current state.

The concept of forward and backward search is popular in the artificial intelligence literature [Barr and Feigenbaum, 1981]. Production systems typically represent two types of problem solving. The forwardchaining approach basically starts from the available information and tries to draw conclusions that are appropriate to the goal(s). Forward chaining is a generalization of the logical operator modus ponens: From $p$ and (if $p$ then $q$ ), infer $q$. Backward chaining, on the other hand, starts from the goal and looks for evidence that supports or contradicts this goal. By using production rules that are in the form of (if condition then action), backward chaining tries to match the action parts of rules with the current goal. It then looks at the left hand side of those rules to determine what conditions would make them "fire," then finding other rules whose action parts conclude these conditions, and so on.

Traditionally, simulation studies have employed the "forward" approach for addressing various production related problems, such as facility design and capacity analysis, schedule analysis, and schedule generation. In all these instances, a current state is assumed and a future state is predicted based on the execution of events and activities over time. This section introduces the concept of backward simulation and contrasts the modeling requirements with the forward simulation approach.

\subsection{Backward Simulation}

Backward search is at the heart of many problem-solving techniques. For instance, mathematical programming techniques include the dynamic programming method [Hillier and Lieberman, 1986]. This method is generally applicable to any problem that can be broken down into a sequence of stages and states. In general, this solution procedure begins by finding the optimal policy for the last stage and moves backward, stage by stage. The concept of backward search is also popular in the artificial intelligence literature in such strategies as backtracking, backward reasoning and backward chaining [Barr and Feigenbaum, 1981]. As with dynamic programming, these concepts do not incorporate the passage of time. However, the PERT/CPM project planning technique [Moder, et. al., 1983] does involve the passage of time. A forward and backward pass through a network described as time delays (activities) and events is executed to determine activity slack and float. This knowledge is used to manage the scheduling of large-scale project activities such as bridge construction. 
Traditional scheduling packages for discrete part production utilize some form of forward and/or backward scheduling. When forward scheduling, the scheduler starts with the known order release dates and determines corresponding completion dates of each order based on expected lead times. Backward scheduling is essentially the reverse of forward scheduling. Beginning with the due date of each individual order, this method works backward to determine the desired start dates. Material requirements planning (MRP) logic is basically an extension of the backward scheduling concept. The MRP logic also incorporates the vertical and horizontal dependencies inherent in a bill-of-material structure [Orlicky, 1975]. The MRP approach is perhaps the most popular production planning technique used in discrete parts manufacturing.

The implementation of backward scheduling using object-oriented programming is reported by Jain [1990]. Jain suggests that some intelligence can be employed when infeasible backward schedules are generated by shifting the schedule forward in time using idle time intervals on machines until it becomes feasible. However, object-oriented paradigms that employ a frame representation do not have the full representation capabilities of simulation modeling. For this reason, it is noted that resource contention (i.e., queueing) is not typically represented by these approaches.

The backward scheduling concept has also been extended to simulation modeling. With backward simulation, the idea is to start with the goal state and then simulate the passage of time backward to the initial state. In this context, the goal state corresponds to due date satisfaction, and the initial state corresponds to a specific order release schedule. The advantage of the simulation approach, of course, is the powerful modeling capabilities that may be employed to capture accurately resource levels, operational characteristics, and subsequently, the impact of queueing delays. Although a large number of simulation-based backward scheduling applications are not published, the few that do exist are discussed below.

A two-level capacity loading and operational scheduling tool for a rolling mill was developed by Gelders and Van Steelandt [1980]. The operational model forward simulates the orders in process and backward simulates the orders not yet in process. The actual schedule generation is via a priority rule that is based upon available capacity and float time. A relatively simple product structure is generally assumed by this industry, and therefore, the analyst is able to evaluate float time for jobs that are assigned to key resources. In a similar manner, backward and forward models were developed for scheduling operations in the aircraft assembly industry by Pope, et. al. [1990] and for a group technology cell application by Yunk [1981]. Basically, the forward approach is used to schedule follow-on operations for work-in-process. The backward approach uses the inverse of the routing sequence to generate a trace of material flow given the end point requirements.

Simulation is also being used as an experimental method to evaluate certain characteristics of backward schedules versus forward schedules. In a simple job shop environment, Mejtsky [1985] determined that backward simulation can find non-zero delay schedules that are better than schedules generated with forward simulation that assume zero-delay schedules. After experimenting with a small contrived job shop setting, Kim [1987] suggests that backward sequencing is better when job structure resembles assembly operations (i.e., in tree) and worse when job structure is disassembly (i.e., out tree).

The concepts behind backward simulation-based scheduling for a discrete parts environment are similar to forward simulation. Typical requirements for such a system include: a simulation model of the system that describes how jobs progress through the system backward; part routings that indicate workcenter visitations, set up times, and process times; a bill of material structure for each end item; and an order due date schedule (e.g., the master production schedule). Orders are released according to an end item master plan and journey through the facility as dictated by their inverted part routing. Hence, orders enter through the exit station and exit through the enter station. The order start dates that are required to achieve the desired due dates can then be determined. The bill-of-materials (BOM) ensure that the vertical and horizontal dependencies are obeyed. The simulation model ensures that any limitations, exceptions, and assumptions are obeyed.

In both research and practice, the implications of these results are limited to the specific problem studied. In all of the published literature reviewed on the subject of backward simulation for discrete parts production, the inferences drawn are limited.

\subsection{Backward Simulation Comparison}

Discussions on simulation-based forward scheduling can be found in Sturrock and Higley [1987] and Larsen and Alting [1990]. A simple comparison of the backward simulation and forward simulation approach is presented below. As expected, backward simulation for detailed scheduling is similar, in many respects, to forward simulation for detailed scheduling. 


\section{Similarities}

1. Both approaches use the same discrete simulation language. Hence, the time advance and event list processing mechanisms employed by the simulation are the same.

2. Both models are deterministic. Hence, neither approach requires the use of a random number generator or a random variate generator.

3. Both models may incorporate some level of slack. The idea is that as demand uncertainty increases over time, so should planning slack.

4. Resource utilization statistics are valuable to indicate load, per planning period.

5. File management is required due to the enormous amount of order information.

6. Standard animation, error checking, and diagnostic tools are valuable model building and presentation tools.

\section{Differences}

1. Part flow through the facility is reversed in the backward simulation (i.e., the part routing is reversed). An assembly operation in the forward pass would be a disassembly operation in the backward pass.

2. The concept of queueing is not intuitive in the backward simulation.

3. The bill of material is processed bottom-up during the forward simulation and top-down during the backward simulation

4. Simulation languages do not allow updating time backward, therefore, the time axis must be reversed for the backward simulation.

5. The backward simulation model cannot make use of traditional sequencing rules to achieve the same effects as the forward simulation model. Each set of rules must be designed for the particular model objectives.

6. The initial conditions and objectives are different.

7. Simulation-based forward schedules are always achievable, though they may result in excessive order tardiness. Simulation-based backward schedules are not achievable if an order release is scheduled for a time prior to the current time.

8. The backward approach does not incorporate workin-process into the schedule. If work-in-process is significant, and not incorporated, the resulting schedule may be infeasible.

In short, the backward simulation approach to detailed simulation has found some applications in industry. It is important to remember, though, that no matter how powerful a scheduling method is, how well it performs depends a great deal upon how good the plan is. Most finite scheduling applications assume a component release plan that is generated from a planning system based on traditional MRP logic. This paper contends that an MRP-based plan may be infeasible to begin with. The next section introduces the BACKSIM approach; an approach to generating component release plans that is based on a backward and forward simulation model.

\section{BACKSIM APPROACH}

The function of generating component release schedules in traditional discrete manufacturing shops is typically performed by a commercial or in-house MRP-based software package. Over the years, numerous enhancements have been made to this type of resource management software in an attempt to make the product more functional, more effective, and more marketable. Quite ironically, though, the heart of the MRP-logic is the same as it was in the 1960's.

The concept of planning for dependent demand items based on the forecasts of independent demand items has proven to be invaluable for coordinating planning activities. But at the time when MRP was conceived, the value of material resources far outweighed that of capital resources. This resulted in a planning system that focused on material and left capacity as a secondary consideration. A capacity requirements planning (CRP) module is executed after the MRP and generally requires manual interaction. In fact, it is not uncommon to find a production manager who only uses MRP as a long-term loading tool because the manager is neither comfortable, nor confident with the CRP module.

Regardless of the extent to which the aggregate plan anticipates and incorporates shop floor conditions, the detailed scheduling and control activity is responsible for converting the aggregate plan into a feasible production schedule. This conversion process will be most successful when detailed schedules are developed in conjunction with the aggregate plan and where resource contention and shop-floor constraints are accurately represented. In practice, planning and scheduling are often disjointed, leading to schedules based on past experience rather than on consideration of future system conditions. The integration of aggregate and detailed scheduling activities would allow for the effective utilization of resources, control of work-in-process, and reporting of both job status and job completion times.

An alternative approach to planning for component release is presented in this section. The basic idea is illustrated in Figure 2. Most discrete part manufacturers today have some form of master production scheduling (MPS) system. The MPS basically dictates what enditems are due when. These systems may use a variety of techniques (e.g., forecasting, rough-cut capacity 
planning, resource planning, production planning, and demand management) to generate demand requirements from multiple demand sources (e.g., direct customer orders, warehouse replenishment, after market and service parts).

\begin{tabular}{|c||c|c|}
\hline FUNCTION & EXISTING & PROPOSED \\
\hline \hline Create Demand & MPS & MPS \\
\hline Plan for Production & MRP & $\begin{array}{l}\text { Simulation-based } \\
\text { planning }\end{array}$ \\
\hline Schedule & Finite Scheduling & $\begin{array}{l}\text { Simulation-based } \\
\text { scheduling }\end{array}$ \\
\hline
\end{tabular}

Figure 2: Integrated Planning and Scheduling Framework

The MPS is used by the MRP logic to determine component release dates (times) for all dependent demand items. The MRP logic consists of basic rules for converting the production schedules for higher-level items, BOM information, stock status, and other input information into a time-phased plan for production, or procurement, of each item. These rules are grouped under four topics:

1. determination of gross requirements

2. determination of net requirements

3. lot sizing (economic order quantity calculations)

4. off setting by planned lead time

In practice, three major shortcomings are associated with this fundamental MRP logic:

1. MRP fails to take into account the real life capacities of production resources on the factory floor, and it cannot adequately represent decisions made on the shop floor, thus producing unrealistic plans by which to schedule.

2. Planned lead times are not dependent on the current shop standards. Planned lead times are generally insensitive to shop load, shop capacity, order lot size, and order priority.

3. The MRP module, lacking any execution-mode capabilities, relies on a finite scheduling package to generate detailed operation schedules (or dispatch lists) for distribution to area supervisors.

In the proposed approach, the MPS is still utilized to dictate demand for the customer goods. The generation of order release plans is the function of the simulationbased approach (BACKSIM). The BACKSIM approach consists of two functions: simulation-based backward pass and simulation-based forward pass. If the forward pass simulation model is at the appropriate level of detail for scheduling, then the component release plan generated from BACKSIM is suitable for implementation. Otherwise, a more detailed scheduling pass can be made to generate dispatch lists for primary and secondary resources. In this manner, component release plans are more likely to be feasible and the platform exists for a clean integration of the planning and scheduling tasks.

Numerous "finite scheduling" software packages have been developed to serve as the scheduling arm of MRP systems. The power of these sophisticated scheduling packages is often dampened because the order release plan feeding their system may be infeasible to begin with. Also, and perhaps more importantly, the MRP system and scheduling system use facility models based on different concepts and are susceptible to inconsistent assumptions. For instance, MRP systems generate plans that ignore capacity constraints. MRP cannot incorporate multiple resource types and special resource schedules or operator logic. A good finite scheduling package may be capable of incorporating this logic. If this type of logic is significant, it should be considered at the planning stage. Hence, an integrated planning and scheduling approach, based on the same shop floor assumptions, could have substantial advantages.

\section{THREE-STEP APPROACH}

Planning and scheduling for production, using the backward simulation concept, may require up to three "models," or world views, of the facility. A summary of this concept is illustrated in Figure 3. The first model executes the backward pass which serves to smooth the production requirements based on available capacity. The backward pass is executed with a deterministic simulation model that incorporates the key capacities and logic of the shop floor. End-items are released at their due dates and flow through the simulated shop backward, based on their job routings. When a job is completed, if it is a component that requires no assembly, its component release priority is recorded. Otherwise, all of its children components are released and follow the same logic.

The backward pass deals mainly with the primary resources. It will attempt to smooth production by considering future demand and current orders, as well as current and anticipated shop conditions. The forward pass will ensure that a feasible schedule is generated by integrating the future order releases with the current open orders. The forward pass model is a deterministic model 


\begin{tabular}{|c|c|c|c|c|c|}
\hline STEP & PURPOSE & INPUT & OUTPUT & $\begin{array}{l}\text { SIMULATION } \\
\text { MODEL }\end{array}$ & HORIZON \\
\hline Backward Planning Pass & $\begin{array}{l}\text { - Production smoothing } \\
\text { - Generate release } \\
\text { priorities }\end{array}$ & - MPS & $\begin{array}{l}\text { - Component release } \\
\text { priorities }\end{array}$ & $\begin{array}{l}\text { - Backward } \\
\text { - Aggregate } \\
\text { - Deterministic }\end{array}$ & $\begin{array}{l}\text { - MPS planning } \\
\text { horizon }\end{array}$ \\
\hline Forward Planning Pass & $\begin{array}{l}\text { - Integrate open orders } \\
\text { - Confirm scheduled } \\
\text { releases }\end{array}$ & $\begin{array}{l}\text { - Component release } \\
\text { priorities } \\
\text { - Open order status }\end{array}$ & $\begin{array}{l}\text { - Component release } \\
\text { schedule }\end{array}$ & $\begin{array}{l}\text { - Forward } \\
\text { - Aggregate } \\
\text { - Deterministic }\end{array}$ & $\begin{array}{l}\text { - MRP planning } \\
\text { period }\end{array}$ \\
\hline Detailed Scheduling Pass & $\begin{array}{l}\text { - Generate detailed } \\
\text { dispatch list }\end{array}$ & $\begin{array}{l}\text { - Component release } \\
\text { schedule }\end{array}$ & - Dispatch list & $\begin{array}{l}\text { - Forward } \\
\text { - Detailed } \\
\text { - Deterministic }\end{array}$ & - Per shift \\
\hline
\end{tabular}

Figure 3: Three-Step Approach to Backward Planning

that includes, at least, the primary resources. The future pass execution results in a feasible order release schedule. This schedule may be suitable for distribution to the shop floor if it incorporates enough detail. Otherwise, a scheduling pass is made to provide detail to the dispatch list.

The backward pass should generally extend as far out as the MPS planning horizon to ensure that all anticipated demand is considered. The forward pass should generally extend out to the end of the planning period. Although, the order release schedule for shop floor distribution (e.g., the dispatch list) need only be generated for the near-term horizon. For instance, a rolling-time horizon, $t$, can be defined as the largest value for which the probability of no major disruptions occurring during time, $t$, is very small. Any significant deviations from the schedule make the schedule obsolete, thus requiring another dispatch list generation cycle.

An illustration of the backward and forward planning passes is presented in Figure 4. In this illustration, a multi-level BOM is assumed for part number 1 . Part 1 is assembled from parts 2 and 3. Part 2 is assembled from parts 4,5 , and 6 . Parts $3,4,5$, and 6 originate as raw material. Parts $1,2,3,4,5$, and 6 each have a unique part routing that specifies operation number, workcenter number, set up times, process times, and move times.

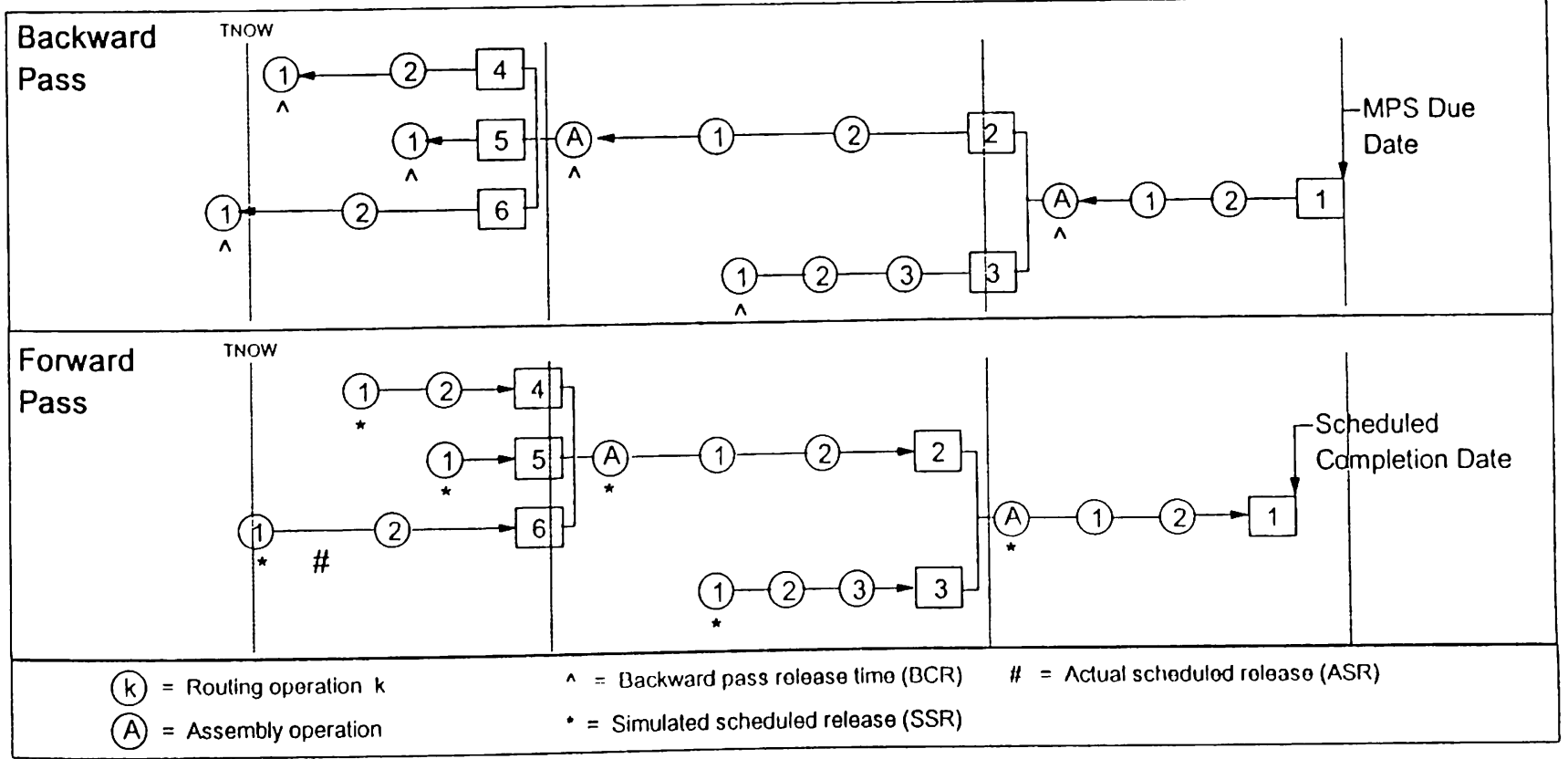

Figure 4: Backward and Forward Planning Pass 


\section{Backward Planning Pass}

The backward pass essentially starts each end item at the MPS due date and sends it through the shop based on the backward sequencing of the routing. For instance, the last operation in the backward pass is the first operation in the forward pass. Parts will queue for the required resources and process based on deterministic standards. After the part completes its last operation, it will either be disassembled or sent to raw material storage. For instance, part 1 (being an assembly) will generate components 2 and 3 . Components 2 and 3 will then flow backward through the simulation model according to their routings.

Depending upon the amount of slack built into the backward pass model, the component release priority might resemble an early start or late start date. During the backward pass, the quickest manner in which a part can be routed through the facility is if it has a high priority and if it does not incorporate any backward pass slack into its move/set up/run process times. In this case, the component release priority would be analogous to a late start time. In contrast, if the part does not have a high priority and if slack is built into the move/set up/run process times, then the component release priority will approach an early start time.

There is a ready time associated with each component in the BOM that originated as either a purchased part or a raw material. The ready time is simply that time in which the part is ready for release to the first operation (i.e., the raw material or purchased part is available). A component cannot be released prior to its ready time. The actual early start time for a part would be equivalent to what has been termed the ready time.

In summary, the backward simulation planning pass consists of determining order release dates from order due dates based on finite capacities and on actual (simulated) component and assembly lead times. These lead times, in this case, will depend on shop load, shop capacity, order lot size, and order priority. This backward explosion extends down to the "leaf" components. Some level of slack is incorporated into this plan based on the level of open orders that exist, as well as on the level of uncertainty in the system. The release time of all leaf components is established during the backward pass as the maximum of backward start time and order ready time. The release dates established during the backward pass are then provided as input into the forward stochastic operational model.

\section{Forward Planning Pass}

The forward pass simulation model integrates current work-in-process (i.e., open orders) with future order releases to ensure that the component release schedule is feasible. The integration of current work-in-process (WIP) is important since the WIP places a high priority demand on the constrained resources. The forward pass uses a component release strategy to calculate a simulated scheduled release (SSR) for every order, product and component triplet, as follows:

$\mathrm{SSR}=\operatorname{MAX}(\mathrm{BCR}, \mathrm{RDY})$.

where:

$\mathrm{BCR}=$ the backward pass component release time for each order, product, and component triplet.

$\mathrm{RDY}=$ ready date for each order, product, and component triplet.

The forward release offset acts as slack for the forward pass. When the job is released to the simulation model at time SSR, it may or may not begin processing immediately, depending upon available capacity. The actual scheduled release time (ASR) is when the job actually seizes its first resource minus the forward release slack. For instance, in Figure 1, the backward pass release time (BCR) for component 6 occurs prior to the current time, TNOW. Assuming that the component ready time is the current time (i.e., $\mathrm{RDY}=\mathrm{TNOW}$ ), then the forward pass will push the simulated release time (SSR) for component 6 to the current time. The actual scheduled release time (ASR) is the time at which the component actually starts processing minus some slack for flexibility. ASR is the time specified on the dispatch list for shop floor distribution.

Outside of resource utilization, there may be no desire to collect statistics or generate reports during the backward or forward planning passes. Dispatch lists are generated during the forward schedule model. The importance of the backward pass is not to evaluate performance measures, but rather to set good priorities for the forward scheduling pass. How well it does this is not apparent until the performance measures of the forward pass can be evaluated.

\section{Detailed Scheduling Pass}

As mentioned before, the detailed scheduling pass is necessary when the forward planning pass does not incorporate all of the detail required for schedule generation. For instance, since only primary resources are generally considered during the backward and forward pass, the scheduler may desire to generate a dispatch list for the secondary resources (e.g., tools, fixtures, operators, etc.). 


\section{CONCLUSION}

A primary advantage of the backward simulation-based planning approach is that it determines release dates based on shop congestion. The shop congestion is caused by firm plans as well as open orders (WIP) already released to the system in a prior period but not yet completed. Also, the lead times experienced by each component are a function of the component routing, lot size, priority, as well as the shop load, capacity, and operational rules and assumptions.

Integrated backward/forward simulation-based planning and scheduling is an approach that shows promise for discrete parts make-to-order environments that experience frequent changes to the product mix and/or frequent product line introductions. Just like every other approach, there are certain environments that may be more conducive to this type of planning and scheduling. The modeling capabilities of a simulation language make simulation a superior tool for integrating planning and scheduling functions in the production environment. Dynamic lead time estimates, accurate resource contention and capacity considerations, and powerful modeling capabilities such as modeling routing dependencies and job dependencies are just some of the advantages of such a tool.

For over twenty years, practitioners have been trying to make effective use of material requirement planning (MRP) systems. The planned lead time concept has motivated blatant misuse of safety stocks and safety lead times. With the strong surge towards contemporary manufacturing philosophies (i.e., just-in-time, agile manufacturing, synchronous manufacturing, work cells, etc.), the time is ripe for seriously considering a new approach. A simulation-based planning package would provide an excellent means of determining component release schedules based on realistic shop floor assumptions.

\section{REFERENCES}

Balci, O., Sadowski, R. P., Nance, R. E., Proceedings of the 1990 Winter Simulation Conference, New Orleans, Louisiana, December, 1990.

Barr, A. and Feigenbaum, E. A., The Handbook of Artificial Intelligence Volume I, Addison-Wesley Publishing Company, Inc., 1981.

Davis, D. A., Sturrock, D. T., Pegden, C. D., Sadowski, R. P., and Watson, E. F., "A Simulation-Based RealTime Decision-Support Tool for Manufacturing," Small Business Phase I, National Science Foundation, NSF Award Number, ISI-8960804, 1990.

Gelders, L. F. and Van Steelandt, F. V., "Design and Implementation of a Production Planning System in a
Rolling Mill: A Case Study." AIIE Transactions, March 1980.

Hillier, F. S. and Lieberman, G. J., Introduction to Operations Research, Holden-Day, Inc., 1986.

Jain, S., Barber, K., Osterfeld, D., "Expert Simulation for On-Line Scheduling." Communications of the ACM, October, 1990, Vol. 33, No. 10.

Kim, Y., "On the Superiority of a Backward Approach in List Scheduling Algorithms for Multi-Machine Makespan Problems." International Journal of Production Research, 1987, Vol. 25, No. 12, 17511759.

Larsen, N. E. and Alting, L., "Requirements to Scheduling Simulation Systems." Summer Computer Simulation Conference, The Society of Computer Simulation, B. Surcek, J. McRae (eds.), Alberta, Canada, July 16-18, 1990.

Mejtsky, G. J., "Backward Simulation and MultipleObjective Optimization of Job Shop Scheduling with Zero Tardiness and Minimum Makespan." The Proceedings of the 1985 Summer Computer Simulation Conference, Chicago, Illinois, July 22-24, 1985

Moder, J. J., Phillips, C. R., Davis, E. W., Project Management with CPM, PERT and Precedence Diagramming, 3rd edition, Van Nostrand Reinhold Company, New York, N.Y., 1983.

Nelson, B. L., Kelton, W. D., Clark, G. M., Proceedings of the 1991 Winter Simulation Conference, Phoenix, Arizone, December, 1991.

Orlicky, J. Material Requirements Planning, McGrawHill Book Company, 1975.

Pope, D. N., Courney, S. R., Autrey, J. P., Hsu, H., Uses of Simulation in Scheduling Aircraft Manufacturing Operations." IIE Integrated Systems Conference and Society for Integrated Manufacturing Conference Proceedings, 1990.

Richardson, S., "Finite-Capacity Scheduling with FACTOR," IIE Integrated Systems Conference and Society for Integrated Manufacturing Conference Proceedings, 1990.

Rothenberg, J., Narain, S., Steeb, R., Hefley, C., and Shapiro, N. Z., Knowledge-Based Simulation: An Interim Report, The RAND Corporation, N-2897DARPA, 1989.

Sturrock, D. T. and Higley, H. B., "Scheduling with Simulation: A Case Example." World Productivity Forum and 1987 International Industrial Engineering Conference Proceedings.

Swain, J. J., Goldsman, D., Crain, R. C., Wilson, J. C., Proceedings of the 1992 Winter Simulation Conference, Arlington Virginia, December, 1992. 
Yunk, N. T., "Computer Simulation Applied to Shop Floor Scheduling and Control," Fall Industrial Engineering Conference Proceedings, 1981.

\section{AUTHOR BIOGRAPHIES}

EDWARD F. WATSON currently conducts research and consulting activities with the Services Division at Systems Modeling Corporation. His primary responsibilities are to spearhead and manage large scale capacity and scheduling related projects. He received his bachelors degree at Syracuse University and his masters at Penn State. Mr. Watson is completing his doctorate work in the Industrial Engineering Department at Penn State.

DEBORAH J. MEDEIROS is an Associate Professor in the Department of Industrial Engineering at Penn State University. Her research interests include design, analysis and control of manufacturing and material handling systems, and manufacturing applications of coordinate measuring machines. She holds a BSIE from the University of Massachusetts and an MSIE and Ph.D. from Purdue University.

RANDALL P. SADOWSKI is currently vice president of Systems Modeling Corporation in charge of consulting and user-education services. He was previously on the faculty at Purdue University and at the University of Massachusetts. He received his bachelors and masters degrees from Ohio University and his Ph.D. from Purdue. Dr. Sadowski's research interests are in manufacturing and production systems with emphasis on modeling, control, and applied scheduling. 